\title{
Microwave dielectric heating of non-aqueous droplets in a microfluidic device for nanoparticle synthesis
}

\section{Citation}

Koziej, Dorota, Caspar Floryan, Ralph A. Sperling, Allen J. Ehrlicher, David Issadore, Robert Westervelt, and David A. Weitz. 2013. “Microwave Dielectric Heating of Non-Aqueous Droplets in a Microfluidic Device for Nanoparticle Synthesis." Nanoscale 5 (12): 5468. doi:10.1039/ c3nr00500c.

\section{Published Version}

doi:10.1039/c3nr00500c

\section{Permanent link}

http://nrs.harvard.edu/urn-3:HUL.InstRepos:26516707

\section{Terms of Use}

This article was downloaded from Harvard University's DASH repository, and is made available under the terms and conditions applicable to Open Access Policy Articles, as set forth at http:// nrs.harvard.edu/urn-3:HUL.InstRepos:dash.current.terms-of-use\#OAP

\section{Share Your Story}

The Harvard community has made this article openly available.

Please share how this access benefits you. Submit a story.

Accessibility 


\title{
Microwave dielectric heating of non-aqueous droplets in a microfluidic device for nanoparticles synthesis
}

\author{
Dorota Koziej, ${ }^{a, b, *}$ Caspar Floryan, ${ }^{a}$ Ralph A. Sperling, ${ }^{a}$ Allen Ehrlicher, ${ }^{a}$ David Issadore, ${ }^{c, d}$ Robert \\ Westervelt, ${ }^{a}$ and David A. Weitz,
}

\section{Received (in $X X X, X X X)$ Xth $X X X X X X X X X 20 X X$, Accepted $X$ th $X X X X X X X X X 20 X X$ DOI: $10.1039 / b 000000 x$}

\begin{abstract}
We describe a microfluidic device with an integrated microwave heater specifically designed to dielectrically heat non-aqueous droplets using time-varying electrical fields with the frequency range between 700 and $900 \mathrm{MHz}$. The precise control of frequency, power, temperature and duration of the 10 applied field opens up new vistas for experiments not attainable by conventional microwave heating. We use a non-contact temperature measurement system based on fluorescence to directly determine the temperature inside a single droplet. The maximum temperature achieved of the droplets is $50^{\circ} \mathrm{C}$ in $15 \mathrm{~ms}$ which represents an increase of about $25^{\circ} \mathrm{C}$ above the base temperature of the continuous phase. In addition we use an infrared camera to monitor the thermal characteristics of the device allowing us to 15 ensure that heating is exclusively due to the dielectric heating and not to other effects like non-dielectric losses due to electrodes or contacts imperfection. This is crucial for illustrating the potential of dielectric heating of benzyl alcohol droplets for the synthesis of metal oxides. We demonstrate the utility of this technology for metal oxide nanoparticle synthesis, achieving crystallization of tungsten oxide nanoparticles and remarkable microstructure, with a reaction time of $64 \mathrm{~ms}$ a substantial improvement 20 over conventional heating methods.
\end{abstract}

\section{Introduction}

The properties of nanoparticles differ from those of their counterpart bulk form due to confinement effect caused by their ${ }_{25}$ size, structure, and shape. ${ }^{1}$ To optimize these properties for specific applications it is useful to carry out synthetic reactions with small volume and low cost. Microfluidic reactors are valuable tools for this application, due to their ability to rapidly dose reagents and to create homogenous mixtures on the scale of

\footnotetext{
${ }^{a}$ School of Engineering and Applied Sciences, Department of Physics, Harvard University, Cambridge, MA 02138, USA. Tel: 01617496 9788; ${ }^{b}$ ETH Zürich, Department of Materials, Laboratory for Multifunctional Materials, 8093 Zurich, Switzerland. Fax: 0414633 15 45; Tel: 0414 4633 6055; E-mail koziejd@mat.ethz.ch

${ }^{c}$ Center for Systems Biology, Massachusetts General Hospital, Boston, MA 02114, USA

${ }^{d}$ Department of Bioengineering, School of Engineering and Applied Science, University of Pennsylvania, Philadelphia, Pennsylvania, USA

$\dagger$ Electronic Supplementary Information (ESI) available: optical images of the microfluidc device; measurements of dependence of the microwave frequency on temperature of benzyl alcohol droplets; summary of dielectric properties of common solvents; a quasi-static electrical field simulation; TEM, HRTEM and SEAD of nanoparticles washed with ethanol. See DOI: 10.1039/b000000x
}

30 microns. $^{2-7}$ Microfluidic systems can be used either in continuous flow or segmented flow through the use of droplets. The uses of segmented flow reactors, in comparison with their continuous counterparts, allow nanoliter volumes of reaction solutions to be independently controlled with precise reaction conditions, such as 35 heat, mass transfer rates, and temperature.. ${ }^{2-7}$ The ability to separate and control the stages of nucleation and growth is a unique aspect of microfluidic synthesis. These properties found broad application in fabrication of metal, ${ }^{8}$ III-VI semiconductors 9 and polymeric ${ }^{10}$ nanoparticles with defined size and structure. ${ }_{40}$ Recent developments have been directed toward scaling out of the processes by adding reactors working in parallel. ${ }^{11,12}$ Despite enormous progress in using microfluidic reactors for the synthesis of nanoparticles, crystallinity of metal oxide nanoparticles has only been demonstrated in systems where the reactants are 45 maintained at high temperatures for at least 1 to 30 minutes. This can be accomplished through the use of a resistive heater, oil bath or oven, which heats either the entire device, or just the collection tube. $^{13,14}$ An alternate means of efficiently increasing the temperature is to heat the sample alone. This can be done through 50 the use of microwaves, which selectively and directly heats the sample itself. ${ }^{15-17}$ Microwave heating can significantly increase the rate of synthesis of metal oxide nanoparticles in bulk, reducing the reaction time from hours to minutes. ${ }^{18}$

Recently, we have shown that microwave heating can be 
integrated into droplet based microfluidic chips, enabling rapid (< $30 \mathrm{~ms}$ ) heating to temperatures $30^{\circ} \mathrm{C}$ above the base temperature. ${ }^{19}$ In this previous study, we heated water at $f=$ $2.4 \mathrm{GHz}$, utilizing its large dielectric resonance loss at microwave 5 frequencies. Several novel device geometries and materials have been employed to fabricate the efficient transmission of microwave power to samples on microfluidic chips. ${ }^{20,21}$ Because the research on microfluidic-microwave reactor has been driven by its potential application for DNA amplification or cell lysis,

10 the state-of-the-art devices are dedicated to heating of water in frequency range between 2.45 and $15 \mathrm{GHz}$. However, water is not a suitable solvent for the low temperature synthesis of many inorganic metal oxides in crystalline states. Instead solvents such as benzyl alcohol are utilized, since in bulk it both enables rapid

15 microwave synthesis and efficient crystallization at relatively low reaction temperatures. ${ }^{18}$ Despite widespread use of microwave heating in the synthesis of nanoparticles, using microwave heating has never been done with a microfluidic device and the advantages of this technique has not been explored.

20 In this paper we describe a microfluidic dielectric-heating device, operating at $700-900 \mathrm{MHz}$, which is capable of precisely heating the non-aqueous solvents used for nanoparticles synthesis, for instance benzyl alcohol, n-butanol and ethylene glycol. ${ }^{22-26}$ Label-free IR temperature imaging provides quantitative 25 information about the dielectric heating of the benzyl alcohol droplets, heat transfer from the droplets, and non-dielectric losses. Additionally we measure with high temporal resolution the dielectric heating of the benzyl alcohol droplets by fluorescence imaging. To demonstrate the utility of this approach, 30 we synthesize crystalline tungsten oxide nanoparticles in benzyl alcohol using our chip. Typically tungsten oxide synthesis leads to a $\mathrm{WO}_{3} \cdot \mathrm{nH}_{2} \mathrm{O}$ product in the form of platelets independent of the heating method. ${ }^{27,28}$ Tungsten oxides nanoparticles have been widely used materials for application in gas sensing, water 35 splitting, electrochromic windows. In addition, unconventional applications of tungsten oxide nanoparticles are for high Tc superconductors, field emission displays and optical recording devices. ${ }^{29-32}$ Therefore, we choose this material to show the feasibility of microwave synthesis on a microfluidic chip.

\section{${ }_{40}$ Materials and methods}

\section{Device}

The microfluidic device is fabricated using standard soft lithography. The PDMS channels are $50 \mu \mathrm{m}$ high. The device was plasma bonded to a thin glass slide $(0.13 \mathrm{~mm})$, the channels were

45 functionalized with Aquapel and baked for 20 minutes at $60^{\circ} \mathrm{C}$. The electrodes are fabricated by applying a low-melting solder fill technique previously described. ${ }^{33}$ The electronics to generate the microwave power at $700-900 \mathrm{MHz}$ are assembled similar to the electronics for $3 \mathrm{GHz}$ frequency. ${ }^{19}$ The microwaves are ${ }_{50}$ generated with a voltage-controlled oscillator (ZX95-1200W+, Mini-Circuits) and are amplified with a power amplifier (ZHL211-8 Mini-Circuits). The quasi-static simulation of the electrical field between the electrodes is performed with Ansoft, Maxwell software.

\section{${ }_{55}$ Calibration of temperature measurements}

We use rhodamine $\mathrm{B}(\mathrm{RhB})$ and rhodamine $110(\mathrm{Rh} 110)$ at the concentration of $0.1 \mathrm{mmol}$ and measure the emission spectra on a Leica TCS SP5 confocal microscope equipped with heating stage (Warner Instruments). The dyes are excited at $488 \mathrm{~nm}$ and the ${ }_{60}$ emission is examined by scanning in $5 \mathrm{~nm}$ steps between 500 and $650 \mathrm{~nm}$ at different temperatures. The two dyes have different temperature-dependent spectra when excited at $488 \mathrm{~nm}$ allowing the individual signal to be separately measured and the temperature of the sample determined by calibration.

\section{${ }_{65}$ Single droplets temperature measurement set-up}

The detection setup consists of a $50 \mathrm{mM}, 488 \mathrm{~nm} \mathrm{cw}$ laser coupled into the backport of a Motic AE-31 fluorescence microscope. The laser is focused by a 40x, NA 0.85 objective into the flow channel of the microfluidic device, which is heated by 70 microwaves. The emitted fluorescence is collected by the same objective and passed through a series of dichroic mirrors at the back of the microscope. Photomultiplier tubes behind bandpass filter detect the light at 536/40, data acquisition is carried out on a PC with a FPGA card from National Instruments and a LabView 75 program.

\section{Thermal IR imaging}

The temperature profile of the device is measure with an IR camera SC 5600 FLIR equipped with InSb detector using a frame rate $100 \mathrm{~Hz}$. The $0.13 \mathrm{~mm}$ thick glass slide is partially transparent 80 to infrared; therefore we cannot image completely through the glass and measure temperature directly in the channel. In the detector spectral range $(3-5 \mathrm{~mm})$ the transmittance of glass lies between $90-40 \%$.

\section{Chemicals}

85 Anhydrous benzyl alcohol ( $\geq 99 \%)$, tungsten hexachloride ( $\geq 99.9 \%)$, rhodamine B $(97 \%)$, and rhodamine $110(\geq 99.0 \%)$, oxalyl chloride (98\%), Poly(propylene glycol)-blockpoly(ethylene glycol)-block-poly(propylene glycol) bis(2aminopropyl ether), anhydrous dichloromethane (99.8 \%) were 90 supplied by Sigma-Aldrich, Fluorinert Electronic Liquid FC-40 (FC-40 oil) and HFE-7100 by 3M, polyethylene oxide, M.W. 100 000 by Alfa Aesar, Krytox 157 FSH by Miller-Stephenson,

All chemicals were used without further purification. SU8 3050 was purchased from MicroChem, polydimethylsiloxane (PDMS) 95 prepolymer and curing agent - Sylgard 184- from Essex Brownell, indium alloy Ind19 (52In, 32.5 Bi, 16.5 Sn) 0.020 " diameter, from Indium Corporation, Aquapel from Pittsburgh Glass Works, LLC.

\section{Synthesis in microfluidic device}

100 Tungsten hexachloride $(20 \mathrm{mg})$ was added, in an oxygen- and water-free atmosphere, to anhydrous benzyl alcohol $(3 \mathrm{ml})$. The light blue solution is directly transferred to a glass syringe. The surfactant to stabilize benzyl alcohol droplets in FC-40 oil is synthesized according following published procedure. ${ }^{34}$ Then, $1051 \mathrm{wt} \%$ of surfactant is dissolved in FC-40 and the resulting solution is filtered and transferred to a glass syringe. The liquids are injected into the channels using syringe pumps from Harvard Apparatus. The experiments are performed at a constant flow rate of benzyl alcohol of $20 \mathrm{ml} / \mathrm{h}$ and the oil at $120 \mathrm{ml} / \mathrm{h}$, unless 110 otherwise stated. After the heating of the droplets in the microfluidic-microwave device the solution turns from transparent bluish to slightly yellowish. In the control 
experiments, the reaction solution is heated in the CEM Explorer laboratory microwave in the $10 \mathrm{~mL}$ vessel $(7.5 \mathrm{~mL}$ reaction solution) at $60^{\circ} \mathrm{C}$ for $1-30$ minutes and at $120^{\circ} \mathrm{C}$ for 10 minutes. We did not observed formation of precipitate even after the 5 solution was centrifuged for several hours at the speed of $15,000 \mathrm{rpm}$.

\section{Synthesis of poly(perfluoropropylene glycol)-PEG}

Krytox 157 FSH is a perfluorinated polyether with a carboxylic 10 group at one end. The carboxylic group is converted to the acid chloride by reacting it with an excess of oxalyl chloride. After evaporating off the excess of oxalyl chloride, a diamine PEG is added. The acid choride of the fluorinated block reacts with the amino groups of the PEG, forming di- and triblock molecules. 15 Unreacted PEG is separated by centrifugation; evaporation of the solvent yields the surfactant that can be used without further purification.

\section{Materials characterization}

Transmission Electron Microscope (TEM) and high-resolution 20 Transmission Electron Microscope (HR-TEM) investigations are performed on a Philips Tecnai F30 at $300 \mathrm{kV}$. Scanning Electron Microscope (SEM) studies are performed on a Zeiss FESEM Supra55VP at $5 \mathrm{kV}$. The droplets drip directly from the collection tube of the PDMS device onto carbon-coated copper grids (TEM) ${ }_{25}$ or alumina stub (SEM) were immediately vacuum dried. X-ray powder diffraction (XRD) is measured at Panalytical XPert Pro Diffractometer $(45 \mathrm{kV}, 40 \mathrm{~A})$ equipped in $\mathrm{X}^{\prime}$ 'cellerator detector and fixed slits. The repeated-scans at 2 Theta from 10 to $60^{\circ}$ are merged to improve signal-to-noise ratio.

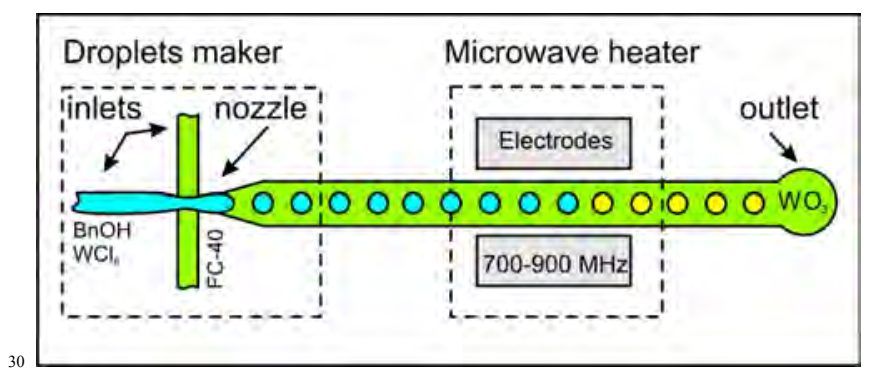

Fig. 1 Schematic of the microfluidic device. The flow-focusing droplet maker is followed by a microwave heater. The benzyl alcohol droplets are rapidly heated using microwaves of $862 \mathrm{MHz}$ frequency (7.5 V tuning voltage). Each droplet is a micro-reactor for ultra-fast nanoparticle 35 synthesis; one example is tungsten oxide.

\section{Results and discussion}

\section{Device for microwave heating of non-aqueous solvents}

Our droplet based micro-reactor is fabricated from PDMS using soft lithography and consists of a droplet maker followed by a 40 microwave heater as schematically illustrated in Fig. 1 and shown in SI Fig. S1, The droplet maker is in the flow focusing geometry, with inlets that are each $20 \mathrm{~mm}$ wide and a nozzle that smoothly widens from $20 \mathrm{~mm}$ to $50 \mathrm{~mm}$. Microwave power is locally delivered by indium-alloy electrodes that are directly integrated 45 into the microfluidic device ${ }^{19}, 33$ and are situated $0.5 \mathrm{~cm}$ downstream from the droplet maker. The frequencies of the rotational relaxation process of benzyl alcohol molecules is approximately $850 \mathrm{MHz}{ }^{23}$ In order to efficiently generate heat the frequency of the heater should approximately matches this of ${ }_{50}$ benzyl alcohol. At $2.45 \mathrm{GHz}$ the molecules of benzyl alcohol are not able to follow the electrical field oscillation and behave like less-polar solvent. Therefore a device designed to heat water with microwave heating using frequency $2.45 \mathrm{GHz}(400 \mathrm{~mW})$ is not efficient for heating of benzyl alcohol. Conventional laboratory 55 microwave ovens compensate for the low energy absorption of non-aqueous solvents at $2.45 \mathrm{GHz}$ by using high output power. Instead for our experiments, we construct an inexpensive microwave source that can supply up to $800 \mathrm{~mW}$ at the frequency range between 700 and $900 \mathrm{MHz}$. Although, we test this device at 60 different frequencies with benzyl alcohol (see ESI Fig. S2), it can be also used without further modification with other common non-aqueous solvents for nanoparticles synthesis. ${ }^{24-26,} 35$ For example 1-haxanol, 1-butanol, 2-propanol, glycerol and diethylene glycol all have higher relaxation times ( $t$ ) than that of ${ }_{65}$ water and therefore their maximal dissipation factors (d) are below $2.45 \mathrm{GHz}$ (Table 1, ESI). The microwave power is controlled by varying the peak-to-peak voltage of the amplifier, and the frequency is controlled by a voltage controlled oscillator. We match the impedance of the electrodes to that of the source 70 and optimize the electrodes geometry to ensure efficient delivery of microwave power to the droplets; we model the quasi-static electrical field to help optimize the device (see ESI, Fig. S3). As a fluid we use FC-40 3M oil with $1 \mathrm{wt} \%$ Krytox-PEG surfactant $^{34}$ for the continuous phase and benzyl alcohol for the 75 dispersed phase.



Fig. 2 The temperature change of the droplets as a function of the peakto-peak voltage. The red curve represents droplets smaller than the channel, formed at a flow rate of benzyl alcohol 10-20 $\mu 1 / \mathrm{h}$ and flow rate 80 of oil $120 \mu \mathrm{l} / \mathrm{h}$. The black curve represents droplets larger than the height channel, which are thermo formed compressed by the cannel, they are formed at higher flower rates $50 \mu 1 / \mathrm{h}$ and higher flow rate of benzyl alcohol and flow rate of oil at $120 \mu \mathrm{l} / \mathrm{h}$. The baseline temperature is $25^{\circ} \mathrm{C}$.

\section{Temperature within the benzyl alcohol droplets}

${ }_{85}$ To determine the temperature within the droplets we measure the fluorescence intensity of dyes dissolved in the benzyl alcohol. We first calibrate the temperature dependence of the fluorescence intensity of rhodamine B and 110, since little is known about their behavior in benzyl alcohol. ${ }^{36,} 37$ Interestingly, the ${ }_{90}$ fluorescence intensity of each dye decreases linearly with 
temperature; this is unlike the behavior in water where only $\mathrm{RhB}$ is temperature dependent. ${ }^{38,} \quad 39$ The measured temperature sensitivities of $\mathrm{RhB}$ at $575 \mathrm{~nm}$ and $\mathrm{Rh} 110$ at $530 \mathrm{~nm}$ are very similar and are $1.75 \% /{ }^{\circ} \mathrm{C} \pm 0.08 \% /{ }^{\circ} \mathrm{C}$ and $1.74 \% /{ }^{\circ} \mathrm{C} \pm 0.08 \% /{ }^{\circ} \mathrm{C}$, 5 respectively (see ESI Fig. 4 and 5). Further, we measure the fluorescence intensity from both dyes in droplets of benzyl alcohol in the microfluidic chip, and use this to determine the temperature within the droplets when they are heated with the microwaves. Fluorescence is excited by a $488 \mathrm{~nm}, 50 \mathrm{~mW}$ laser

10 that is focused on the droplets when they are in the flow channel, between the electrodes used for the microwave heating. The fluorescence emitted from each individual droplet is collected by a microscope objective and guided through a series of dichroic mirrors to three photomultiplier tubes. We record the

15 fluorescence intensity of many droplets as a function of the peak voltage applied to the microwave heater, and determine the average over one thousand equally-sized droplets. To ensure the accuracy of the results we pick the equally-sized droplets from the whole population of droplets by setting the lower limit of

20 fluorescence detection and the duration of droplets, as shown in the ESI, Fig. 6.

In contrast to long-exposure fluorescence imaging of many drops traveling thorough the microwave heater, ${ }^{40-42}$ this method does not require sophisticated background extraction and perfectly ${ }_{25}$ equally-sized drops The temperature rises linearly with microwave power, which is proportional to the square of the peak voltage applied to the electrodes, as shown in Fig. 2. The maximum temperature of the droplets is $50^{\circ} \mathrm{C}$ which represents an increase of about $25^{\circ} \mathrm{C}$ in $15 \mathrm{~ms}$. Interestingly, when we

Os

$0.01 \mathrm{~s}$

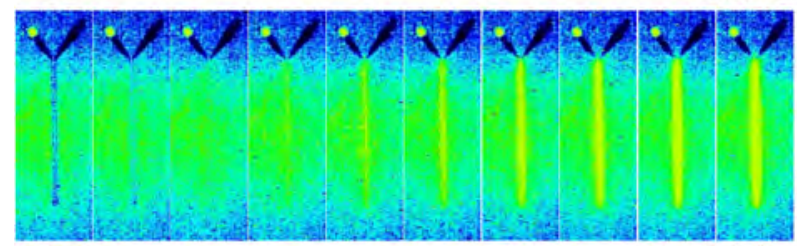

$0.51 \mathrm{~s}$

$5.01 \mathrm{~s}$



$7.51 \mathrm{~s}$

$15.01 \mathrm{~s}$
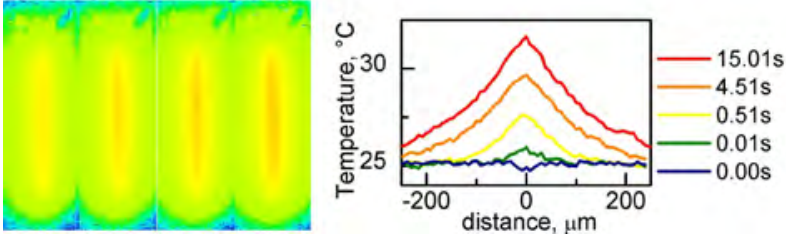

Fig. 3 IR image sequence of microwave heating taken at 100 frames per second. Inset bottom, right - the temperature profile as a function of distance from the center of the channel.

compare the temperature of droplets different in size, we can 35 distinguish two regimes; for droplets smaller than the size of the channel, the slope of the temperature increase with microwave power is steeper and the maximum temperature is higher than that for the larger droplets. Because the larger droplets are deformed by the walls and therefore in close proximity to the PDMS, heat 40 is more efficiently conducted to the bulk of the device; by contrast the carrier oil has a low thermal conductivity $(\kappa \mathrm{FC}-40$ $\left.0.065 \mathrm{~W} \cdot \mathrm{m}^{-1} \cdot \mathrm{C}^{-1}\right)^{43}$ which in case of smaller droplets, effectively prevents dissipation of heat from the droplets into the device. The standard deviation of temperature $\left(\sigma_{\mathrm{T}}\right)$ is equal $1.5^{\circ} \mathrm{C}$ for smaller ${ }_{45}$ droplets, and $0.8^{\circ} \mathrm{C}$ for large droplets. The error in the temperature measurements comes from the variation of the vertical position of droplets smaller than the channel height, which has an impact on focal point and thus on the fluorescence intensity.

\section{${ }_{50}$ Evaluation of dielectric and non-dielectric heating}

There is an ongoing debate whether the enhancement of chemical reactions driven by microwave irradiation is due to rapid heating or whether it is due to selective interaction of electromagnetic field with polar molecules. Our microfluidic - microwave droplet ${ }_{55}$ heater, due to its ability to separate conventional heat transfer mechanisms from dielectric heating ${ }^{44}$, can help shine light onto these questions. To separate the affects of conventional heating from dielectric heating, we utilize infrared imaging to visualize the heat distribution through the device. To directly evaluate the 60 influence of the carrier oil on the heat transfer from the droplets, we measure the temperature distribution on the glass surface of the device using an IR camera. The camera is calibrated to determine the temperature in the images, which is reflected by the color map.

${ }_{65}$ We collect images at the rate of $100 \mathrm{~Hz}$ using an integration time of $2.2 \mathrm{~ms}$, allow us to monitor the change in temperature of the glass surface as the device heats up when a peak voltage of $8.85 \mathrm{~V}$ is first applied to the microwave heater. However, what we essentially measure is the amount of heat vertically dissipated 70 to the substrate from the droplets as the microwave is turned on. The IR images show that the device heats up in the region below which the microwaves are applied over the course of about $15 \mathrm{~s}$; as illustrated in Fig. 3a. We extract the time evolution of the lateral temperature profiles of the heater and we find that the 75 temperature gradient is established on the length of $0.5 \mathrm{~cm}$ when the steady state is reached as shown in the inset of Fig. 3. Even after just $10 \mathrm{~ms}$ the temperature of the glass directly below the microwave heater clearly begins to increase; after $500 \mathrm{~ms}$ we can measure the heat dissipated also in horizontal directions. Steady 80 state conditions are established after $15 \mathrm{~s}$ and the temperature of the device remains constant over several hours of continuous operation. This temperature gradient shows that the benzyl alcohol droplets are hotter than their surroundings, thus verifying dielectric heating. ${ }^{19,45,46}$

${ }_{85}$ To quantitatively determine the heat losses, we measure the temperature of the device when it reaches steady state. We determine the temperature profiles along the channel from the IR 
(a)

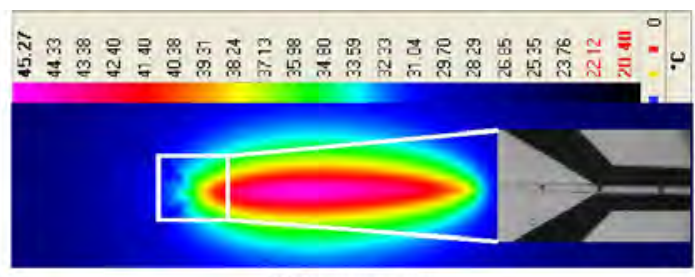

Distance, $\mathrm{cm}$
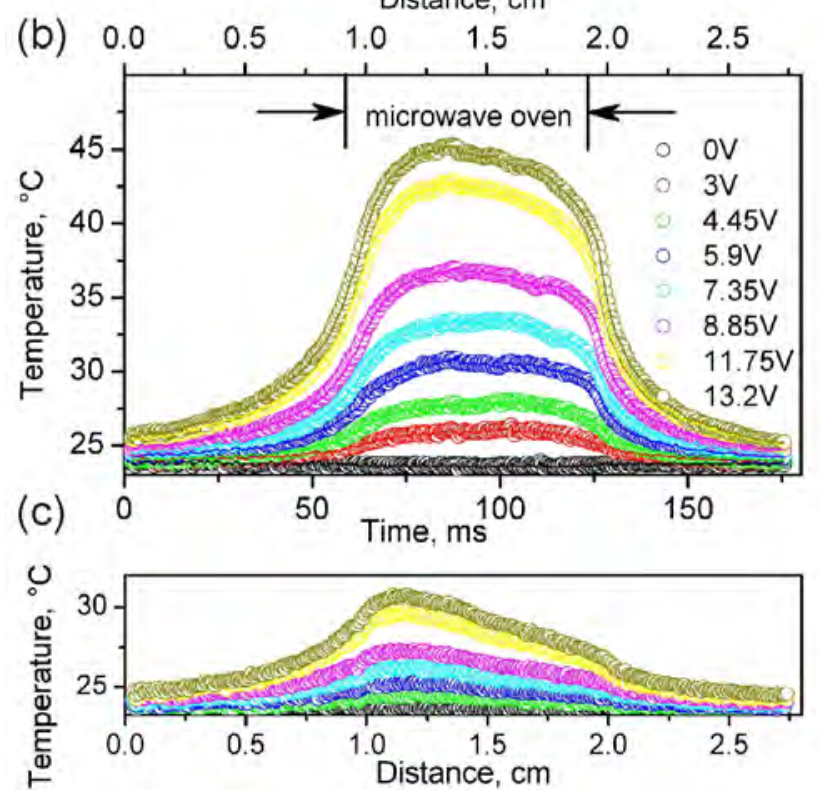

Fig. 4 (a) Two-dimensional temperature map of heat dissipation at the surface of a microfluidic-microwave heater. In the inset shows an optical image of the droplets entering the microwave heater area, (b) temperature profiles along the channel at different peak-to-peak voltages in the presence of microwave-absorbing benzyl alcohol droplets, and (c) corresponding background temperature profiles when only the nonabsorbing FC-40 oil is flowing.

images. We observe an abrupt increase in the temperature at the 10 entrance of the microwave heater and a corresponding abrupt decrease at the exit; the temperature remains constant along the heated area. Adjusting the applied microwave voltage from 0 to 13.2 $\mathrm{V}$ peak-to-peak changes the temperature from 25 to $45^{\circ} \mathrm{C}$ as shown in Fig. 4 a,b. This represents an increase of $20^{\circ} \mathrm{C}$, 15 somewhat less than that in the drops themselves. To differentiate microwave heating of the fluid from resistive heating we remove the benzyl alcohol from the device and flow only non-absorbing fluorinated oil (FC-40). In this case, there is very little temperature change measured, as the device only heat up by 20 maximum of $5^{\circ} \mathrm{C}$ as shown in Fig. $4 \mathrm{c}$; this residual temperature rise can be attributed to traces of resistive heating, which may come from artifacts such as electrode imperfections.

\section{Honeycomb-like microstructure made of nanoparticles}

The ability to rapidly deliver power to the non-aqueous droplets 25 using dielectric heating offers new opportunities to synthesize inorganic materials. To illustrate this potential, we use our microfluidic microwave heater to synthesize tungsten oxide nanoparticles within benzyl alcohol droplets using the synthesis protocol for conventional reaction in oil bath described by ${ }_{30}$ Niederberger et al. ${ }^{27}$ We use the microwave-microfluidic device described above to generate droplets of tungsten hexachloride and benzyl alcohol solution in FC-40 oil. The droplets of ca. $50 \mu \mathrm{m}$

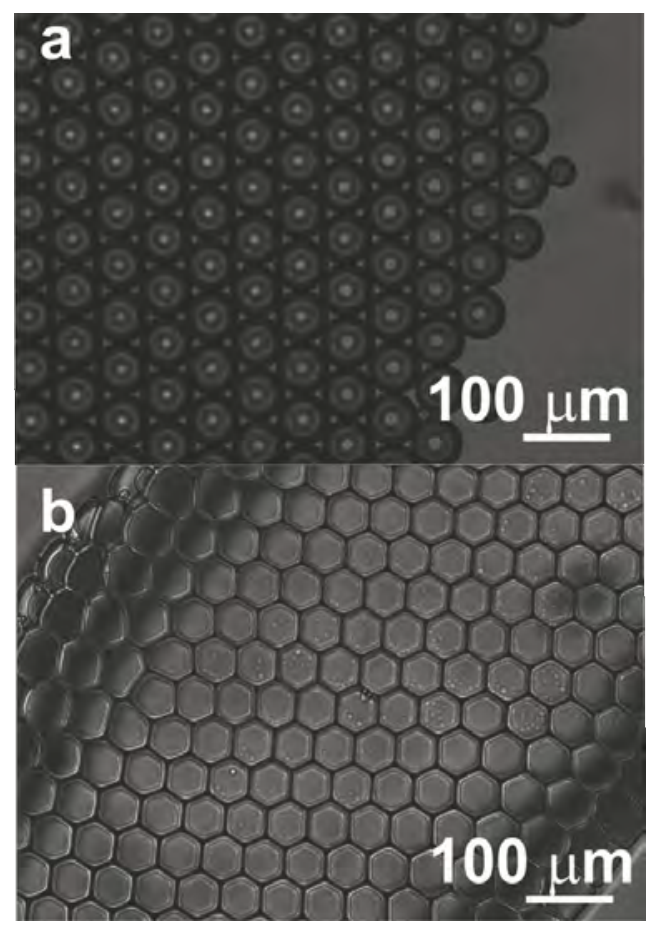

Fig. 5 Optical microscope images of single monolayer of benzyl alcohol 35 droplets: (a) benzyl alcohol droplets immerse in continuous FC-40 phase self assemble onto glass surface, (b) after continuous phase (FC-40) evaporates the droplets are still stable and adapt a honeycomb-like microstructure.

reside in the area exposed to dielectric heating of $50^{\circ} \mathrm{C}$ for $64 \mathrm{~ms}$. 40 An example of droplets and their assembly after evaporation of FC-40 is shown in Fig. 5. After drying in air, and without further washing, the unique honeycomb-like microstructure is formed. If the structure is dried onto the polished Si-wafer in vacuum, the honeycomb-like microstructure is only partially preserved. Most ${ }_{45}$ likely the poor adhesion between the dried droplets and the $\mathrm{Si}$ substrate results in the partial de-attachment of the microstructure under the vacuum (Fig. 6a). A closer look at the dried droplets shows that there is more solid phase collected at the boundaries between the droplets than in the middle of the single droplet. 50 Additionally, wrinkled microstructure inside the droplets is observed (Fig. 6b). TEM images not only confirm the morphology of the dried droplets but also reveal a subtle nanostructure. The darker regions inside the droplets, clearly visible already in the SEM image as wrinkles, and the edges of 55 the droplets consist of assemblies of primary nanoparticles (Fig. 7a-c).

Inside the droplets the individual nanoparticles are observed. HRTEM images deliver the final proofs of their crystallinity (Fig. 7d-f). The size of primary particles does not exceed $3 \mathrm{~nm}$. ${ }_{60} \mathrm{XRD}$ pattern shows broad reflections typical for nanoparticles that complicates an unambiguous identification of the crystal structure. However, the best match among different tungsten oxide hydrates $\left(\mathrm{WO}_{3} \cdot \mathrm{nH}_{2} \mathrm{O}, \mathrm{n}=0,0.33,0.5,1\right.$ and 2$)$ is obtained with orthorhombic tungsten oxide 0.3-hydrate, ICDD Nr. 01-072${ }_{65} 0199$ (Fig. 8). Interestingly, the size of nanoparticles and their structure change if they are washed with ethanol, which can be observed in the sharp reflection in XRD pattern (Fig. 8 b). Also in HR-TEM image individual $2 \mathrm{~nm}$ nanoparticles cannot be distinguished anymore, but rather nanoparticles of $10 \mathrm{~nm}$ and 
their

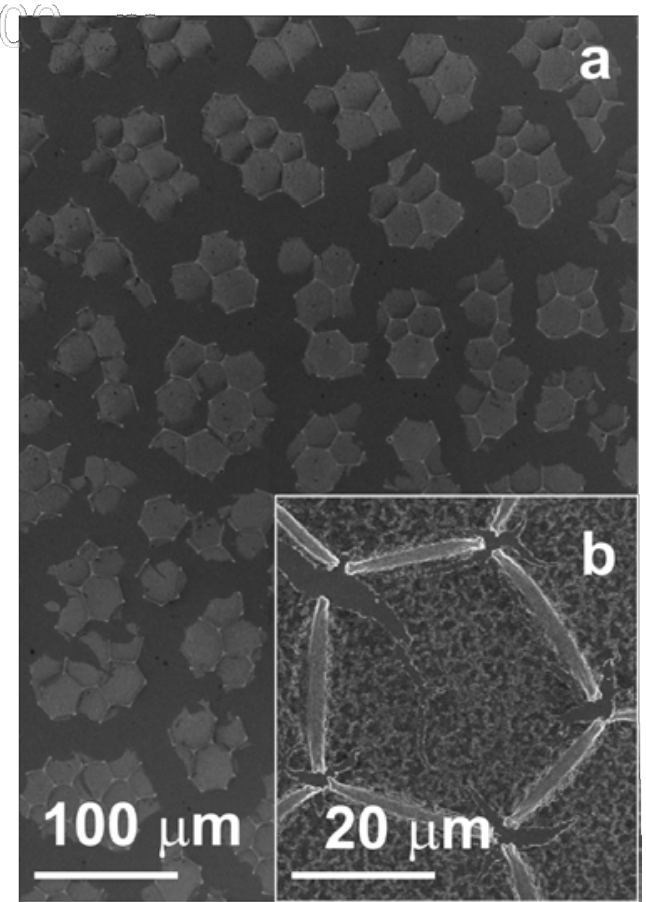

Fig. 6 SEM images of dried droplets at different magnifications, which show (a) a partially preserved honeycomb-like microstructure, and (b) 5 wrinkle-like features inside the droplets.

agglomerates (ESI Fig. S7). The diffraction rings in the corresponding electron diffraction (ED) pattern are characteristic of polycrystalline materials. The sharp reflections in XRD and SEAD patterns can be attributed to the tungstate oxide hydroxide 10 nanoparticles (Fig. 8b).

Likely benzyl alcohol plays not only a role as reaction medium, but also as a capping agent. By washing with ethanol, the organic residuals are removed from the surface of tungsten oxide hydrates. We believe the growth process is driven by the decrease 15 in total system energy by reducing surface energy. Furthermore, heating by irradiation with electron beam accelerates nanoparticles transformation and growth. This is visualized by consecutive recording of the diffraction patterns at the same spot.

We observe a successive transformation of the diffraction rings 20 (SEI Fig. 7a) into well-developed lattice planes (SEI Fig. 8). It is not surprising since benzyl alcohol is known to stabilize small nanoparticles and metastable crystal structures. ${ }^{35,47}$ For instance, we have shown that benzyl alcohol can stabilized the 1-2 nm large $\mathrm{MoO}_{2}$ nanoparticles in hexagonal crystal structure. If ${ }_{25}$ acetophenone is added to the reaction solution, the nanoparticle grow through oriented attachment and transform to the thermodynamically more stable monoclinic phase. Also the role of electron beam irradiation on oriented attachment and growth of nanoparticles was recently visualized by the real-time HR-TEM 30 imaging of growth of $\mathrm{Pt}_{3} \mathrm{Fe}$ and $\mathrm{Au}$ nanoparticles and at the surface of CdSe ${ }^{48,49}$ In case of $\mathrm{Au}$ on CdSe Meyes et al. showed that during irradiation of the sample by the electron beam, the thin shell of $\mathrm{Au}$ at the surface of CdSe nanoparticles evolved into dot-like deposits with high contrast. ${ }^{49}$ The authors suggest that it
${ }_{35}$ can be correlated with the lower degree of coverage with ligands at the surface of $\mathrm{CdSe}$. Whereas in the case of $\mathrm{Pt}_{3} \mathrm{Fe}$ the observations revealed growth of winding polycrystalline nanoparticle chains by shape-directed nanoparticle attachment followed by straightening and orientation and shape corrections 40 to yield single-crystal nanorods. ${ }^{48}$

Finally, we performed the control bulk experiments in the laboratory microwave at $60^{\circ} \mathrm{C}$ and $120^{\circ} \mathrm{C}$ (see ESI Fig. S9). The $\mathrm{XRD}$ pattern of nanoparticles synthesizes at $120^{\circ} \mathrm{C}$ for 10 minutes can be clearly assigned to $\mathrm{WO}_{3} \cdot \mathrm{H}_{2} \mathrm{O}$ crystal structure 45 (ICDD Nr. 00-018-1418). At $60^{\circ} \mathrm{C}$ we did not observed nanoparticles formation at reaction times shorter than 15 minutes. Even though the corresponding XRD pattern shows very broad reflections it can be also assigned to $\mathrm{WO}_{3} \cdot \mathrm{H}_{2} \mathrm{O}$.

The nanoparticles synthesized in the microwave-microfluidic ${ }_{50}$ device adopt $\mathrm{WO}_{3} \cdot 0.33 \mathrm{H}_{2} \mathrm{O}$ crystal structure in contrast to $\mathrm{WO}_{3} \cdot \mathrm{H}_{2} \mathrm{O}$ obtained in our control synthesis in the laboratory microwave and to experiments in oil bath. ${ }^{27,28}$ At the same time the reaction time is decreased from 15 minutes at $60^{\circ} \mathrm{C}$ in bulk laboratory microwave down to only $64 \mathrm{~ms}$ in a microfluidic ${ }_{55}$ device, demonstrating the advantages of this approach. While most previous studies focus on the engineering of the tungsten oxide nanoparticles, wires and their subsequent assembly, ${ }^{30-32}$ our technique offers a route to simultaneously synthesize and assemble the nanoparticles. Recently, a versatile and simple ${ }_{60}$ approach to produce tailor-made hierarchical porous materials was presented. $^{50}$ Using a microfluidic device to produce monodisperse templating droplets of tunable size, the materials with up to three levels of hierarchy were prepared. Adding the microwave-reactor to the microfluidic chip could additionally ${ }_{65}$ give a control on the chemical composition of porous structures.

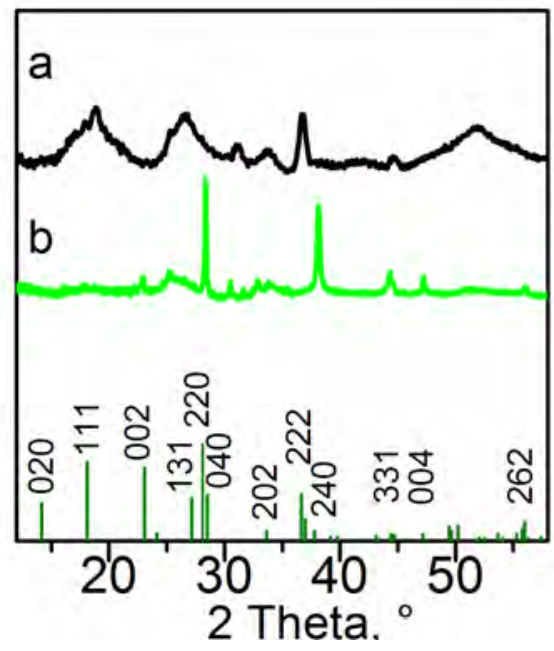

Fig. 8 XRD patterns of reaction product: (a) without washing, (b) after washing with ethanol. The sharp reflection are due to anisotropic grow. Vertical green bars at the bottom: reference pattern of $\mathrm{WO}_{3} \cdot 0.33 \mathrm{H}_{2} \mathrm{O}$, 70 ICDD Nr. 01-072-0199. 

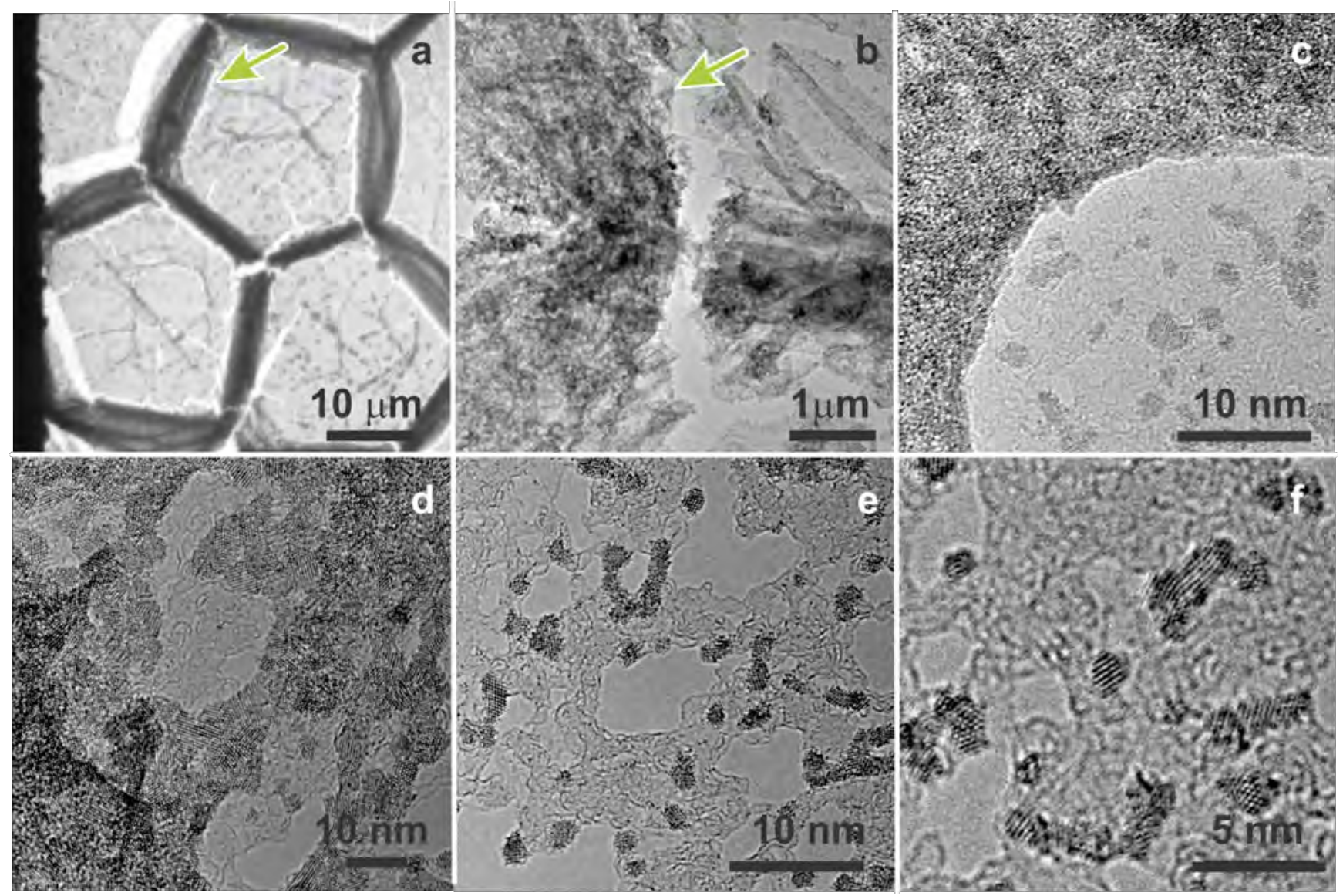

Fig. 7 TEM and HR-TEM images of dried droplets. Green arrow points the spot, which is magnified in the following image (from a to c). The nanoparticles agglomerates do not exhibit preferential crystallographic orientation

\section{Conclusions}

Although microfluidic reactors are often applied to synthesize crystalline nanoparticles, microwave heating has not previously been utilized. Here we show that with microwave heating of $\mathrm{pL}$ sized droplets we can produce appropriate conditions to 10 crystallize inorganic metal oxide nanoparticles. This technique enables future work to alter the concentration of the precursor and the heating time of the droplets, to finely control the assembly of nanoparticles within each droplet. The use of our microfluidic chip leads to drastically reduced synthesis time (from 15 minutes 15 in bulk microwave, down to $64 \mathrm{~ms}$ in microfluidic device). Additionally, our chip has the potential to help address the important issue in microwave driven particle synthesis of distinguishing the effects of non-thermal and thermal effects of microwave irradiation. ${ }^{22}$ The two main constraints in addressing 20 this question are:: the limited compatibility of standard laboratory microwave with spectroscopic equipment; and the low temporal resolution of most of available spectroscopic techniques. With the help of single droplets fluorescence detection and infrared thermal imaging techniques we can ensure that heat is generated 25 only due to dielectric heating of benzyl alcohol. Therefore, in future, a droplet-based microfluidic system can provide a valuable platform for coupling of a dielectric heating and in-situ spectroscopy techniques. Moreover, a unique advantage of the application of spectroscopic technique on a microfluidic chip is 30 the spatial resolution, which can be translated into the enhanced temporal resolution without loss of data quality. ${ }^{51,52}$ Hence, these results reported help assess the utility of microfluidic microwave heating for exceptionally fast inorganic material synthesis.

\section{${ }_{35}$ Acknowledgements}

This work was supported by the NSF (DMR-1006546) the Harvard MRSEC (DMR-0820484), the Swiss National Foundation, projects: PA00P2 129086 and 2-77354-12 and by the Electron Microscopy of ETH Zurich (EMEZ). Martin Süess 40 (EMEZ) help with HR-TEM and Rahel Boehlen support with the soft-litography are greatly acknowledged. DK thank Prof. Markus Niederberger (ETH Zurich) for his enthusiastic support.

\section{Notes and references}

1. A. P. Alivisatos, Science, 1996, 271, 933-937.

45 2. J. Il Park, A. Saffari, S. Kumar, A. Gunther and E. Kumacheva, Annu. Rev. Mater. Res., 2010, 40, 415-443.

3. A. J. deMello, Nature, 2006, 442, 394-402.

4. A. Abou-Hassan, O. Sandre and V. Cabuil, Angew. Chem., Int. Ed., 2010, 49, 6268-6286. 
5. E. M. Chan, A. P. Alivisatos and R. A. Mathies, J. Am. Chem. Soc., 2005, 127, 13854-13861.

6. S. A. Leung, R. F. Winkle, R. C. R. Wootton and A. J. deMello, Analyst, 2005, 130, 46-51.

5 7. I. Shestopalov, J. D. Tice and R. F. Ismagilov, Lab Chip, 2004, 4, 316-321.

8. Y. J. Song, H. Modrow, L. L. Henry, C. K. Saw, E. E. Doomes, V. Palshin, J. Hormes and C. S. S. R. Kumar, Chem Mater, 2006, 18, 2817-2827.

10 9. E. M. Chan, A. P. Alivisatos and R. A. Mathies, Journal of the American Chemical Society, 2005, 127, 13854-13861.

10. S. Q. Xu, Z. H. Nie, M. Seo, P. Lewis, E. Kumacheva, H. A. Stone, P. Garstecki, D. B. Weibel, I. Gitlin and G. M. Whitesides, Angewandte Chemie-International Edition, 2005, 44, 724-728.

15 11. A. Aimable, N. Jongen, A. Testino, M. Donnet, J. Lemaitre, H. Hofmann and P. Bowen, Chem Eng Technol, 2011, 34, 344 352.

12. W. Ehrfeld, V. Hessel and H. Lowe, Microreactors: New Technology for Modern Chemistry, Wiley-VCH2000.

20 13. A. M. Nightingale, S. H. Krishnadasan, D. Berhanu, X. Niu, C. Drury, R. McIntyre, E. Valsami-Jones and J. C. deMello, Lab on a Chip, 2011, 11, 1221-1227.

14. A. Abou-Hassan, O. Sandre and V. Cabuil, Angewandte ChemieInternational Edition, 2010, 49, 6268-6286.

25 15. B. F. Cottam, S. Krishnadasan, A. J. deMello, J. C. deMello and M. S. P. Shaffer, Lab Chip, 2007, 7, 167-169.

16. A. M. Nightingale, S. H. Krishnadasan, D. Berhanu, X. Niu, C. Drury, R. McIntyre, E. Valsami-Jones and J. C. deMello, Lab Chip, 2011, 11, 1221-1227.

30 17. Z. L. Xue, A. D. Terepka and Y. Hong, Nano Lett., 2004, 4, 2227 2232.

18. I. Bilecka and M. Niederberger, Nanoscale, 2010, 2, 1358-1374.

19. D. Issadore, K. J. Humphry, K. A. Brown, L. Sandberg, D. A. Weitz and R. M. Westervelt, Lab on a Chip, 2009, 9, 1701-1706.

35 20. J. Geist, J. J. Shah, M. V. Rao and M. Gaitan, Journal of Research of the National Institute of Standards and Technology, 2007, 112, 177-189.

21. J. J. Shah, S. G. Sundaresan, J. Geist, D. R. Reyes, J. C. Booth, M. V. Rao and M. Gaitan, Journal of Micromechanics and Microengineering, 2007, 17, 2224-2230.

22. S. A. Galema, Chem. Soc. Rev. , 1997, 26, 233-238.

23. B. Gestblom, Chem. Phys. Lett. , 1980, 74, 333-336.

24. R. Harpeness and A. Gedanken, Langmuir, 2004, 20, 3431-3434.

25. X. Hu, J. Gong, L. Zhang and J. C. Yu, Adv. Mater., 2008, 20, 4845 454850

26. M. Tsuji, M. Hashimoto, Y. Nishizawa, M. Kubokawa and T. Tsuji, Chem.--Eur. J. , 2005, 11, 440-452.

27. M. Niederberger, M. H. Bard and G. D. Stucky, J. Am. Chem. Soc., 2002, 124, 13642-13643.

50 28. I. Olliges-Stadler, J. Stötzel, D. Koziej, M. D. Rossell, J.-D. Grunwaldt, M. Nachtegaal, R. Frahm and M. Niederberger, Chem.--Eur. J., 2012, published online DOI 10.1002/chem.201101514.

29. J. Polleux, A. Gurlo, N. Barsan, U. Weimar, M. Antonietti and M.

55 Niederberger, Angewandte Chemie-International Edition, 2006, 45, 261-265.
30. H. D. Zheng, J. Z. Ou, M. S. Strano, R. B. Kaner, A. Mitchell and K. Kalantar-Zadeh, Adv Funct Mater, 2011, 21, 2175-2196.

31. M. Breedon, P. Spizzirri, M. Taylor, J. du Plessis, D. McCulloch, J. 60 M. Zhu, L. S. Yu, Z. Hu, C. Rix, W. Wlodarski and K. Kalantar-Zadeh, Cryst Growth Des, 2010, 10, 430-439.

32. K. Kalantar-zadeh, A. Vijayaraghavan, M. H. Ham, H. D. Zheng, M. Breedon and M. S. Strano, Chem Mater, 2010, 22, 5660-5666.

33. A. C. Siegel, S. S. Shevkoplyas, D. B. Weibel, D. A. Bruzewicz, A.

65 W. Martinez and G. M. Whitesides, Angew. Chem., Int. Ed. , 2006, 45, 6877-6882.

34. C. Holtze, A. C. Rowat, J. J. Agresti, J. B. Hutchison, F. E. Angile, C. H. J. Schmitz, S. Koster, H. Duan, K. J. Humphry, R. A. Scanga, J. S. Johnson, D. Pisignano and D. A. Weitz, Lab Chip, 2008 8, 1632-1639.

35. G. Garnweitner and M. Niederberger, J. Mater. Chem., 2008, 18, 1171-1182.

36. K. G. Casey and E. L. Quitevis, J. Phys. Chem., 1988, 92, 65906594.

75 37. J. F. Lou, T. M. Finegan, P. Mohsen, T. A. Hatton and P. E. Laibinis, Rev. Anal. Chem., 1999, 18, 235-284.

38. S. Ebert, K. Travis, B. Lincoln and J. Guck, Optics Express, 2007, 15, 15493-15499

39. J. Sakakibara and R. J. Adrian, Exp. Fluids, 1999, 26, 7-15.

80 40. T. Robinson, Y. Schaerli, R. Wootton, F. Hollfelder, C. Dunsby, G. Baldwin, M. Neil, P. French and A. deMello, Lab Chip, 2009, 9, 3437-3441.

41. D. Ross, M. Gaitan and L. E. Locascio, Anal. Chem., 2001, 73, 4117 4123

85 42. R. Samy, T. Glawdel and C. L. Ren, Anal. Chem., 2008, 80, 369-375.

43. http://WWW.solutions.3m.com, $3 M^{\mathrm{TM}}$ Fluorinert $^{\mathrm{TM}}$ Electronic Liquid FC-40, 2011.

44. D. Obermayer, B. Gutmann and C. O. Kappe, Angew. Chem., Int. Ed., 2009, 48, 8321-8324.

90 45. J. Geist, J. J. Shah, M. V. Rao and M. Gaitan, J. Res. Natl. Inst. Stan., 2007, 112, 177-189.

46. J. J. Shah, S. G. Sundaresan, J. Geist, D. R. Reyes, J. C. Booth, M. V. Rao and M. Gaitan, J. Micromech. Microeng., 2007, 17, 22242230.

95 47. D. Koziej, M. D. Rossell, B. Ludi, A. Hintennach, P. Novak, J. D. Grunwaldt and M. Niederberger, Small, 2011, 7, 377-387.

48. H. G. Liao, L. K. Cui, S. Whitelam and H. M. Zheng, Science, 2012, 336, 1011-1014.

49. M. Meyns, N. G. Bastus, Y. X. Cai, A. Kornowski, B. H. Juarez, H. Weller and C. Klinke, J Mater Chem, 2010, 20, 10602-10605.

50. A. R. Studart, J. Studer, L. Xu, K. Yoon, H. C. Shum and D. A Weitz, Langmuir, 2011, 27, 955-964.

51. K. L. A. Chan, X. Z. Niu, A. J. de Mello and S. G. Kazarian, Lab Chip, 2010, 10, 2170-2174.

105 52. H. Oyanagi, Z. H. Sun, Y. Jiang, M. Uehara, H. Nakamura, K. Yamashita, L. Zhang, C. Lee, A. Fukano and H. Maeda, J. Synchrotron Radiat., 2011, 18, 272-279. 